\title{
A dynamic phosphate budget model for a eutrophic lake
}

\author{
Lambertus Lijklema \& Arnoldus H. M. Hieltjes \\ Twente University of Technology, P.O. Box 217, 7500 AE Enschede. The Netherlands
}

Keywords: phosphate, model, lake, internal loading

\begin{abstract}
The relations between the external nutrient loading of lakes, recycling through sediments and the resulting productivity are complicated by feed-back mechanisms, seasonal variations and trends. Simulation is a useful tool for the identification of controlling factors and the assessment of the effects of management measures, supplementary to experimental research. The model variables in our dynamic phosphate budget model include inorganic and organic particulate phosphate and dissolved o-phosphate, in both sediments and overlying water. Sediments may be aerobic or anaerobic, depending on topography, temperature and composition. The major processes described are primary production, mineralisation, sedimentation, adsorption and diffusion. Several model parameters have been estimated directly for Lake Brielle (Netherlands). The sediment dilution rate, the extent of anaerobic conditions and the number and character of adsorption sites are important controlling factors.
\end{abstract}

\section{Introduction; objectives}

The model presented here has been developed for a specific lake in the Netherlands. However, our emphasis will be on the more general aspects of the function of such models, in the study of nutrient cycling in lakes and reservoirs, and the development of a model structure in relation to the objectives of a study and the (lack of) knowledge of a system. The initial function of this phosphate budget model was to serve as a research tool in the study of the internal phosphate loading in Lake Brielle, a closed arm of the sea in the south-west of the Netherlands. The lake has an average depth of $5.5 \mathrm{~m}$ and a maximum depth of about $15 \mathrm{~m}$ in the original tidal stream channels. The length of the lake is $11 \mathrm{~km}$, the maximum width $650 \mathrm{~m}$. The hydraulic residence time can be manipulated; the average value is about 100 days. At the time of the study the external phosphate loading was about $12 \mathrm{~g} \mathrm{P} \mathrm{m}^{-2}, \mathrm{yr}^{-1}$ of which $50 \%$ accumulated in the sediments. During the research, data on water quality, sediment composition and adsorption characteristics, pore water composition and sediment release rates as a function of environmental conditions were obtained.

\section{Model function}

The function of a model can vary from a pure research tool to a management tool. As a research tool it serves as a conceptual frame work for understanding interrelated processes integrating, in a qualitative or (semi-)quantitative way, the relations between the variables representing the system behaviour. Models help to bring about an awareness of the complexity of the system and the identification of areas in which knowledge is lacking and research required. In further stages, models can be tested on their ability to represent the observed phenomena and the assumptions underlying our 


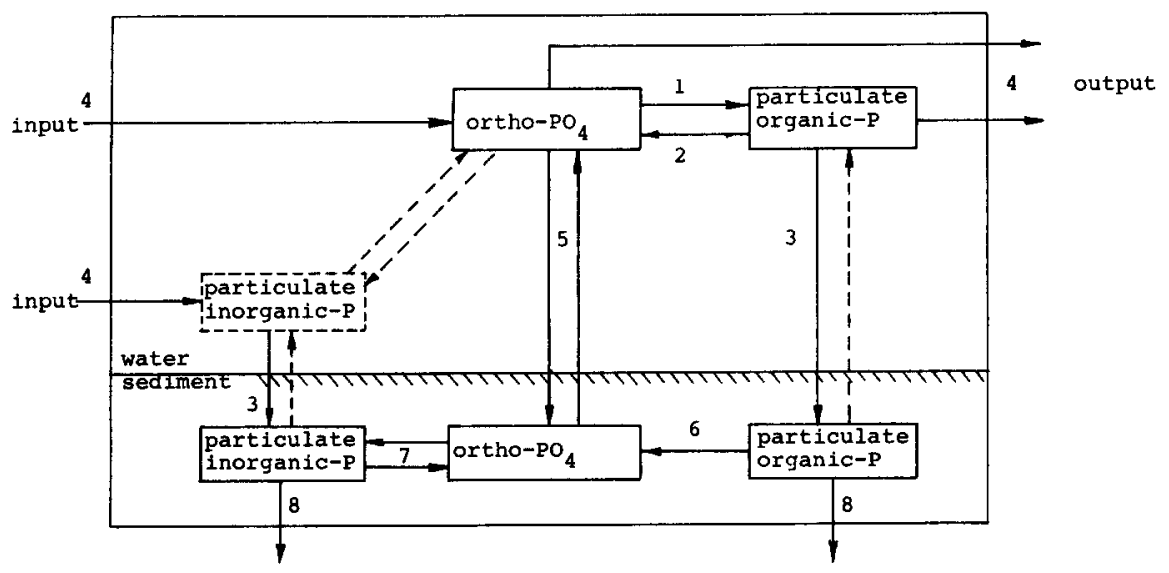

Fig. 1. Definition of model structure.

comprehension of the reality as expressed in the model structure, and on the sensitivity for parameters. A gradual development towards a management tool requires assessment of parameters on the basis of a set of observations and validation based on an independent data set, after which certain predictions and extrapolations for future conditions can be made. The latter function has been tested only in an explorative way in order to identify which factors control the effect of a reduced external load upon the trends in eutrophication.

\section{Model structure}

The model has only three variables, representing different forms of phosphate: particulate inorganic phosphate (PIP), particulate organic phosphate (POP) and dissolved ortho-phosphate (DOP). PIP are inorganic phosphates in insoluble form, e.g. adsorbed on clays, iron coated surfaces, in precipitates etc. POP refers to phosphate in algae, detritus etc. Spatially three compartments are considered: the water body, the littoral sediments and the profundal sediments. All three phases are considered well mixed. Due to erosion and winnowing of littoral sediments and accumulation of fine material in deeper parts of the lake, the lake bottom exhibits a gradual change in grain size distribution, water content, phosphate concentration, P-release rate, redox conditions and probably accumulation rate and mixing depth as well. By differentiating two types of sediments a rough classification of this diversity is attained; the model parameter in this case is the ratio of the surface areas of the two types of sediment. The profundal sediments are also characterised by the possibility of anaerobic conditions. Figure 1 is a representation of the interactions between the variables considered in the model.

\section{Processes}

In this section a short discussion is presented of some of the formulations used in the model, of experimental evidence and of time scales of certain processes. The latter is important because the model aims primarily at the representation of long term changes and hence the time constants of the interactions are related to the degree of detail required in the model equations.

\section{Algal growth, mineralisation ((1) and (2) in Fig. 1)}

These interactions include the uptake of orthophosphate by algae and incorporation in the biomass of the food chain and the reverse - the release of DOP by excretion and mineralisation. The latter also occurs in the sediments (see Fig. 1 (6)). Typically, these processes have a high rate constant (hours, days). Although, ultimately, the goal of this type of study is to understand how the POP level (biomass) can be controlled through a reduction in phosphate loading (and the associated relations are very complex indeed); here, the aim is to obtain a reasonable estimate of the seasonal variation in the rate at 
which DOP is transformed into POP (which is subject to sedimentation and subsequently affects the internal loading). Hence, there is no need for a detailed algal growth model representing daily fluctuations in algal biomass, zooplankton etc. Instead, a simple depth-integrated light-limited growth equation, based on the relation proposed by Smith (1936), has been used. A similar approach has been applied by Lorenzen \& Mitchell (1973). The forcing functions are light and temperature. The coupling with the o-phosphate level is through a simple Monod type concentration dependent growth rate. The rate of mineralisation (2) is first order in POP, and also contains a Monod term in this variable to express the enhancement of mineralisation by grazing etc. at higher levels of biomass.

\section{Sedimentation ((3) in Fig. 1)}

The rate of sedimentation of POP is first order; the proportionality constant is an estimate, based on literature data on settling velocities; $0.3 \mathrm{~m} \mathrm{day}^{-1}$ has been used. This provides a rough estimation of the flux of organic material containing phosphorus to the sediments.

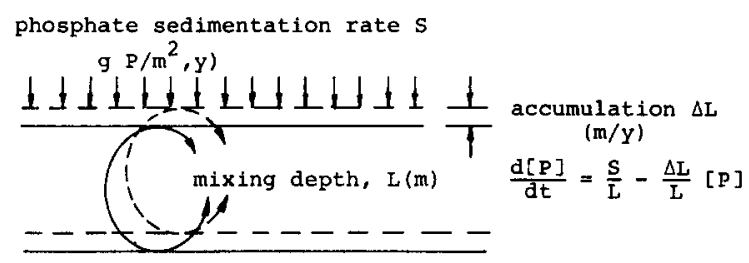

Fig. 2. Dilution and accumulation in sediments.

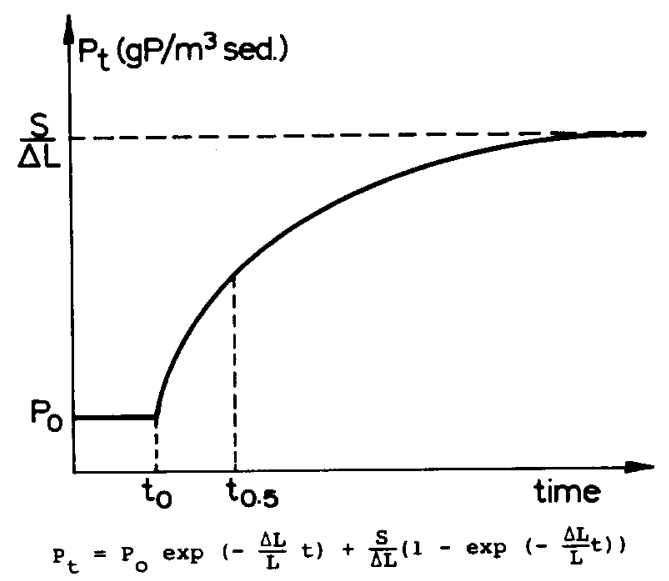

Fig. 3. Enrichment of phosphate in sediment due to a loading rate $S\left(\mathrm{~g} \mathrm{~m}^{-2}, \mathrm{yr}\right)$ from $\mathrm{t}=0$.
An estimate of the distribution of this flux, with respect to the littoral and profundal zones, has been made on the basis of the observed enrichment of phosphorus in deeper sediments. This means that the phosphate sedimentation rate $S$ divided by the annual accumulation $\Delta \mathrm{L}$ is higher in the deeper parts of the lake than in nearshore zones (see Fig. 2). The rate of progress of enrichment is also a function of the sediment mixing depth L; bioturbation depth gives an idea of this value. Values for $S$, $\Delta \mathrm{L}$ and $\mathrm{L}$, for both categories of sediments, were estimated and lake wide retention coefficients for phosphate and suspended solids, were obtained from mass balances. It should be noted that $S / \Delta \mathrm{L}$ controls the equilibrium phosphate concentration in the sediment, whereas $\Delta \mathrm{L} / \mathrm{L}$ controls the rate at which this equilibrium will be attained (see Fig. 3). This time constant will be in the order of decades; hence the sediment dilution rate is an important quantity, which should be obtained by experimental evidence when studying long-term trends in eutrophication and internal loading.

Due to an observed absence of a significant resuspension of sediments containing phosphate, the model does not include a mass balance for PIP. The input of this material, to the lake, is considered to be described by a direct accumulation into the sediments. However, in shallow lakes exposed to wind, the interaction between PIP and DOP may be very important and in such cases the model structure should be changed accordingly (see dotted lines in Fig. 1).

\section{Input, output ((4) in Fig. 1)}

The input is based on measurements of the main sources of water and wastewater. The assumption of an ideal mixed water body is approximately correct due to the short circulation time as compared to the hydraulic retention time. The output of POP and DOP can be calculated on this basis.

\section{Diffusive exchange ((5) in Fig. 1)}

Although more information was available, the release of phosphate by the sediment (as measured in column experiments similar to those described by Fillos \& Biswas (1976) and several other researchers) has been modelled in terms of Fick's law. An empirical value for the diffusion constant was 
obtained from the measured flux, the interstitial phosphate concentration in the sampled top layer and the diffusion distance corresponding to the thickness of this layer. This does not account for the probability that no linear gradient exists in the phosphate concentration, due to enhanced mineralisation of freshly precipitated material in the topmost layer. A more detailed analysis of diffusive release requires the inclusion of terms describing rates of phosphate production, and adsorption and desorption, which cause changes in pore water concentration. This also requires a higher resolution in the techniques for sampling interstitial water (see Brinkman et al. 1982). The calculated values for the effective diffusion coefficient are about $10^{-5} \mathrm{~cm}^{2}$ $\mathrm{s}^{-1}$, a high value, as might be expected. However, the fluxes calculated on this basis are probably not unrealistic because the parameter values are deduced from the experimental data.

One drawback of this approach is that the calculated fluxes are a function only of the environmental conditions (aerobic or anaerobic; see later section) and the phosphate content of the sediment. The well-known brief period, with a high release rate, during the transition from aerobic to anaerobic conditions in the top layer, cannot be simulated by this simplified model approach. This dynamic behaviour is typical for sediments in which iron plays an important role in binding phosphate. Due to dissolution in the anaerobic zone and precipita-

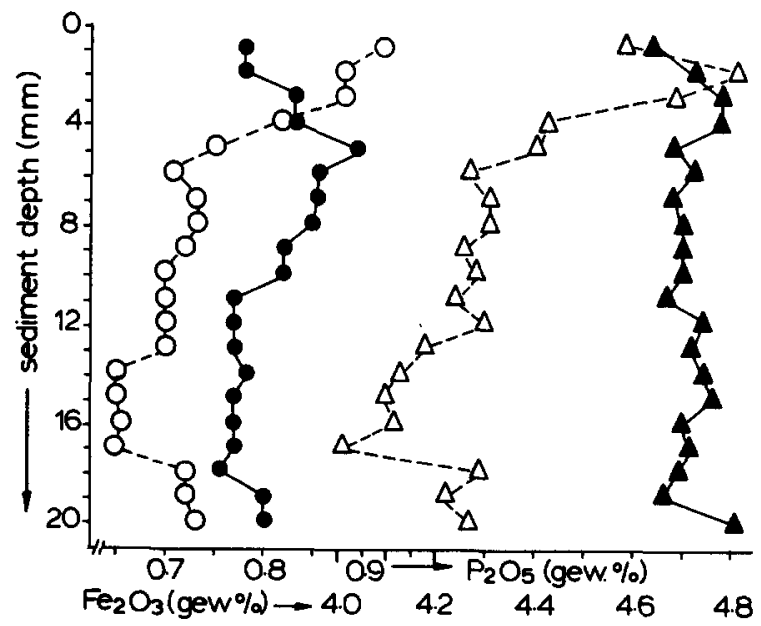

Fig. 4. Content of $\mathrm{P}$ and $\mathrm{Fe}$ as a function of sediment depth: $\mathrm{P}(\bullet)$ and $\mathrm{Fe}(\Delta)$ in sediment with permanent aerobic top layer; $P(O)$ and $\mathrm{Fe}(\Delta)$ in sediment turning anaerobic, three weeks prior to analysis. tion of iron at the interface with the aerobic layer, a zone, enriched with phosphate adsorbed on freshly precipitated iron, migrates gradually towards the sediment-water interface when sediments turn anaerobic during the growing season. The $\mathrm{pH}$ plays an important role in the adsorption and when this shifting zone reaches the interface, where the $\mathrm{pH}$ is much higher, a high flux of phosphate can result (for details of the mechanism, see Lijklema 1980). Experimental evidence of this process is shown in Fig. 4. The sediment with an aerobic top layer is somewhat enriched with phosphate in the top $10 \mathrm{~mm}$, but there is only a slight increase in iron content of the profile. The other sediment profile became anaerobic throughout, for three weeks, following a prolonged period of aerobic conditions. In this latter sediment profile both phosphate and iron are enriched in the top $5 \mathrm{~mm}$. The techniques used involved freezing, grinding and X-ray fluorescense.

\section{Mineralisation in sediments ((6) in Fig. 1)}

This process has two important aspects: it represents the slow production (and seasonal variation) of DOP, and it controls the occurrence of anaerobic conditions. The phosphate release rate is higher when the sediments turn anaerobic, due to the higher phosphate concentration in the interstitial water. The occurrence of anaerobic conditions has been modelled corresponding to a value for the product of temperature and POP. The mineralisation process is first order and rather slow.

\section{Adsorption and desorption ((7) in Fig. 1)}

The equilibrium between PIP and DOP has been formulated as an empirical Langmuir isotherm, based upon experimental data obtained in the laboratory and the field, under both aerobic and anaerobic conditions. The equilibration is thought to be instantaneous. Ultimately, however, it is intended to develop a mathematical model for chemical equilibria which are forced by the slow production of DOP and transport processes (mixing, diffusion, seepage).

\section{Sediment-dilution rate ((8) in Fig. 1)}

The annual burial of a sediment layer of thickness $\Delta \mathrm{L}$ has been discussed already in a previous section. 


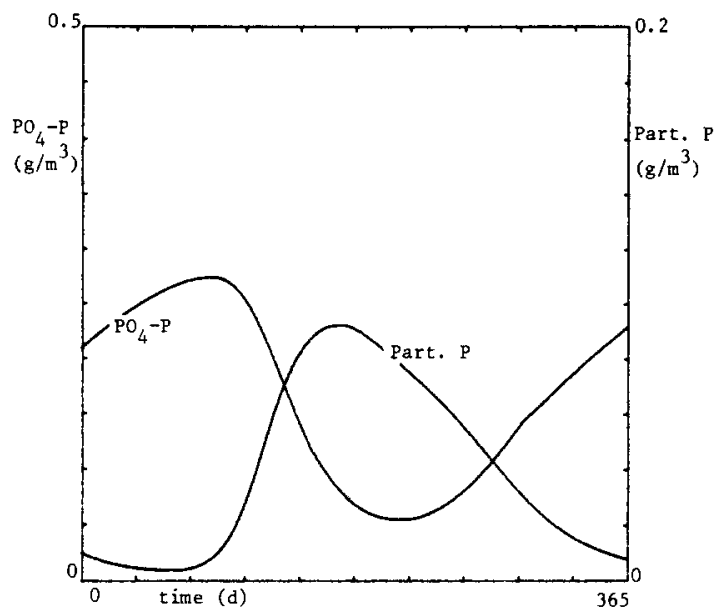

Fig. 5. Annual variation in lake water ortho-phosphate and algal concentration (part. P).

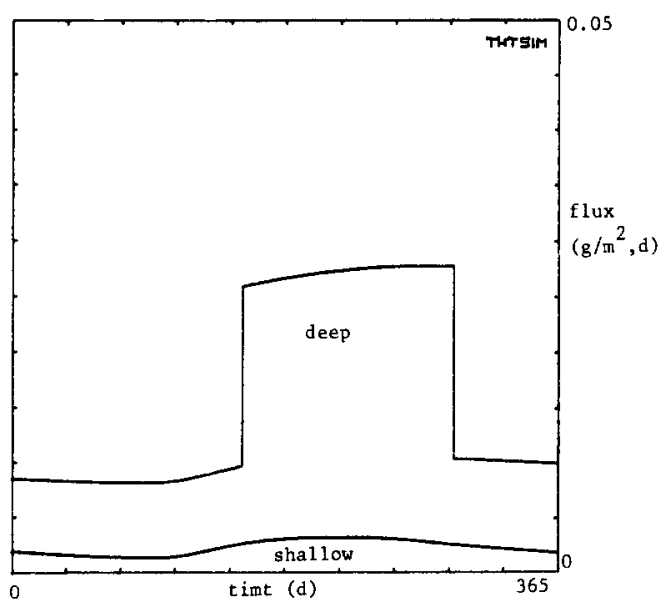

Fig. 6. Typical phosphate fluxes from sediments in shallow and deep zones.

\section{Results and simulations}

Figure 5 shows typical annual variations in DOP and POP in the water, which are in general agreement with field observations. A part of the decrease in DOP during the summer is due to an increased uptake and inclusion of phosphate in algae and other biomass; another part accumulates temporary in the sediments.

Figure 6 shows phosphate fluxes from the two types of sediments but reflects our uncertainty as to the factors controlling the anaerobic conditions. At present, the length is in reasonable accord with

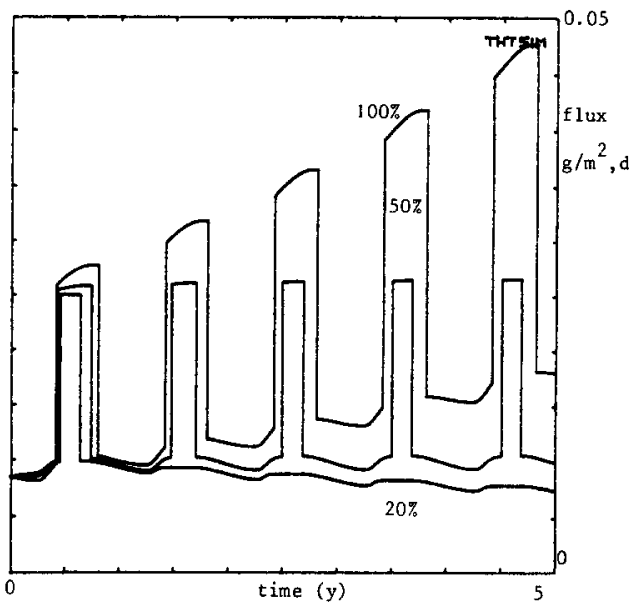

Fig. 7. Simulated response of phosphate flux from deep sediments as a function of external loading.

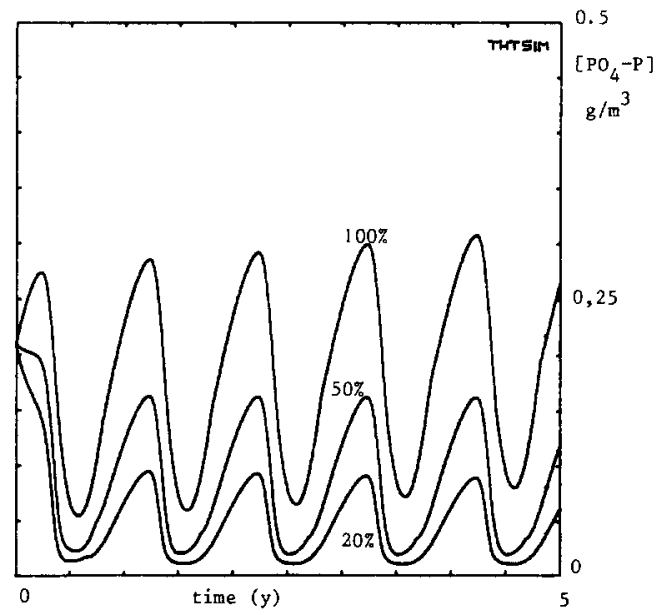

Fig. 8. Simulated response of o-phosphate concentration in lake water as a function of external loading.

observations (the model has been fitted as such); but it is felt that extrapolations from the model could be open to question. The model is rather sensitive in this respect, especially with regard to the rate at which a reduced external loading is reflected in the response of the internal loading. Figure 7 shows the modelled response for loading levels of $100 \%, 50 \%$ and $20 \%$ of present day values. The corresponding phosphate concentrations can be seen (Fig. 8) and are strongly related to the internal loading. The change in the algal concentration (POP) is more or less proportional to the orthophosphate change.

One of the most salient features confirmed by the 


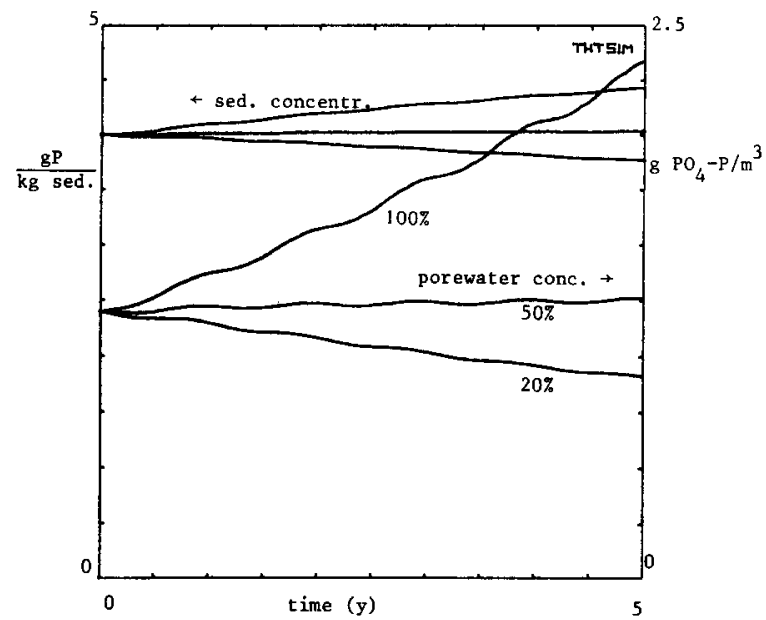

Fig. 9. Content of inorganic phosphate in deep sediments and related equilibrium ortho-phosphate concentration in pore water, as a function of external loading.

model is the 'magnification' of the very slow sediment dilution process in the porewater equilibrium concentration, due to the non-linear character of the adsorption isotherms. Figure 9 presents the sediment concentration and the equilibrium phosphate concentration as simulated for a five year period at three loading levels. At 50\% loading, the present condition will be more or less stable. Due to the exponential attainment of an equilibrium phosphate concentration in the sediments (Fig. 3), the initial response to a change in loading conditions is greatest; and a greater than proportional change in DOP further magnifies the system's response. A qualitative representation of these phenomena is shown in Fig. 10. This figure also indicates one of the questions to be answered before more certainty can be attached to long-term model predictions. The uncertainty concerns the number and character of the adsorption sites in the sediment since a change in loading conditions may also change the shape of the adsorption isotherm, due to some difference in the characteristics of accumulating material and/or a change in the diagenetic processes in the sediments.

Because of difficulties such as these, it seems unlikely that a conceptual chemical equilibrium model could be developed which would be more representative of actual conditions than the present model which uses empirical adsorption isotherms. The necessary experimental information on sediment characteristics for deterministic models, also, will be hard to obtain.

Another observation made during this study is that, in several cases, the effects of changes (e.g. in loading rates) are not always noticeable in the magnitude of state variables (concentrations) but rather in the process rates (fluxes etc.). This also means that model validation should emphasise the measurement of rates rather than concentrations.

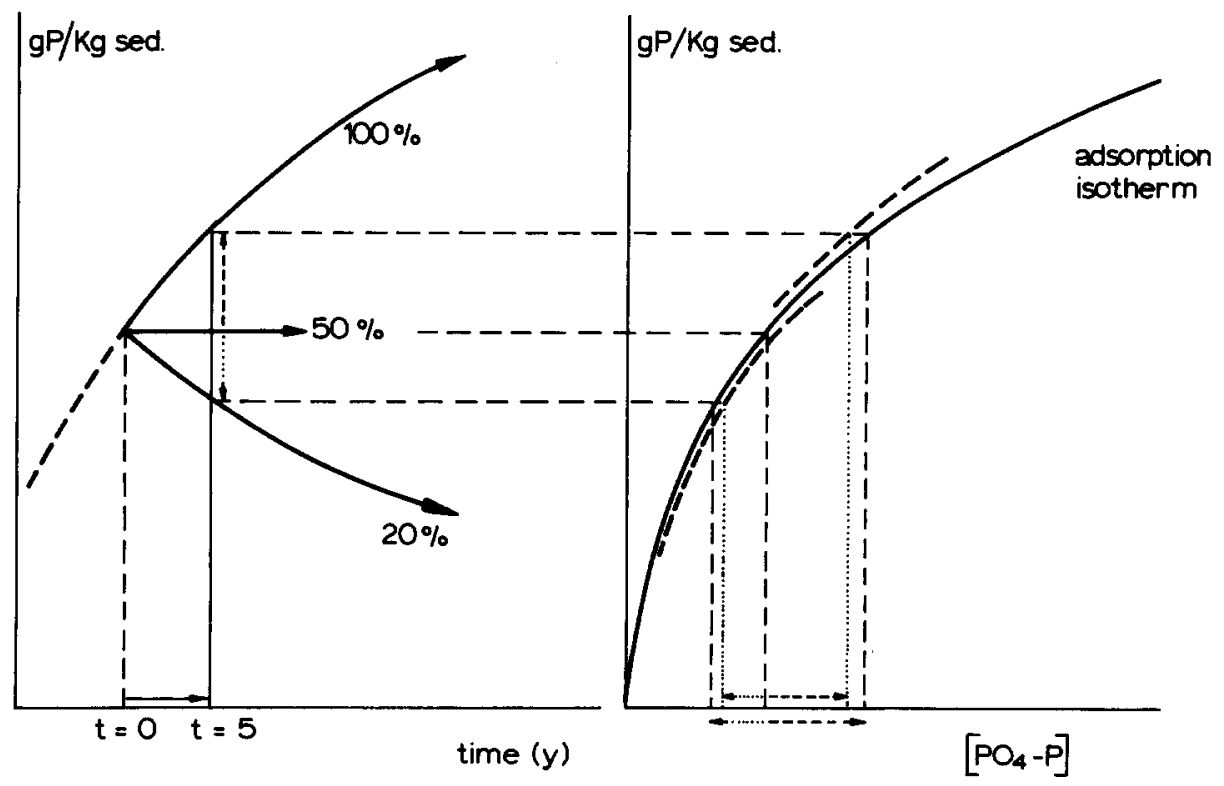

Fig. I0. Response in pore water concentration due to (a change in) external loading. 


\section{Conclusions}

1) A simple phosphate budget model can reproduce the main features of the cycling of phosphate in lakes.

2) The model helps to identify lack of knowledge and research needs.

3) The model predicts that long-term trends in eutrophication are controlled by external loading, the area of sediment surface that may become anaerobic and the time-length of such periods, the sediment dilution rates, and the adsorptive characteristics of the accumulating material.

\section{References}

Brinkman, A. G., van Raaphorst, W. \& Lijklema, L., 1982. In situ sampling of interstitial water from lake sediments. 2nd International Symposium on Interactions between Sediments and Freshwater. (This volume).

Fillos, J. \& Biswas, H., 1976. Phosphate release and sorption by lake Mohegan sediments. J. Env. Eng. Div. ASCE 102 EE2: 239-249.

Lorenzen, M.\& Mitchell, R., 1973. Theoretical effects of artificial destratification on algal production in impoundments. Envir. Sci. Technol. 7: 934-944.

Lijklema, L., 1980. Interaction of orthophosphate with iron(III) and aluminum hydroxides. Envir. Sci. Technol. 14: 537-541.

Smith, E. L., 1936. Photosynthesis in relation to light and carbon dioxide. Proc. nat. Acad. Sci., Washington D.C. 22: 504-511. 